\title{
BRD4 inhibitor JQ1 inhibits and reverses mechanical injury-induced corneal scarring
}

\author{
Mingli Qu', Xiaoping Zhang ${ }^{1,2}$, Xiaoli Hu ${ }^{1}$, Muchen Dong ${ }^{1,3}$, Xiaojing Pan ${ }^{1}$, Jiang Bian ${ }^{1,3}$ and Qingjun Zhou ${ }^{1}$
}

\begin{abstract}
Corneal scarring is characterized by the improper deposition of extracellular matrix components and myofibroblast differentiation from keratocytes. The bromodomain-containing protein 4 (BRD4) inhibitor JQ1 has been shown to attenuate pathological fibrosis. The present study aimed to explore the potential therapeutic effect of JQ1 on mechanical injury-induced mouse corneal scarring and TGF $\beta$-induced human corneal myofibroblast differentiation and the related mechanism. The corneal scarring and myofibroblast differentiation were evaluated with clinical observation and fibrosis-related gene expression analysis. In mice, subconjunctivally injected JQ1 suppressed the initial development and reversed the established progression of corneal scarring, while having no impairment on the epithelial regenerative capacity. BRD4 inhibition with either JQ1 or small-interfering RNA inhibited the differentiation and promoted the dedifferentiation of human corneal myofibroblasts. Moreover, JQ1 attenuated the accumulation of intracellular reactive oxygen species induced by TGF $\beta$ treatment, induced Nrf2 nuclear translocation and activated the expression of Nrf2-ARE downstream antioxidant genes. In conclusion, this study implicates that JQ1 suppresses and reverses corneal scarring through the regulation of BRD4 inhibition and Nrf2-dependant antioxidant induction.
\end{abstract}

\section{Introduction}

Corneal transparency is important for the light transmission to the retina for optimal vision. In human eyes, corneal scarring is the most common result after trauma, infection, or refractive surgery and causes visual impairment ${ }^{1}$. Keratocytes, the major cellular elements in the corneal stroma, remain quiescent and synthesize extracellular matrix (ECM) components to maintain corneal stromal turnover. Following injury, inflammation-induced transforming growth factor $\beta$ (TGF $\beta$ ) activates the transition of corneal keratocytes into fibroblasts and myofibroblasts with the expression of $\alpha$-smooth muscle actin $\left(\alpha\right.$-SMA) as key marker ${ }^{2-5}$. Myofibroblasts rapidly synthesize and secrete redundant ECM proteins, including Collagen I and fibronetin during wound healing, leading

\footnotetext{
Correspondence: Qingjun Zhou (qjzhou2000@hotmail.com)

${ }^{1}$ State Key Laboratory Cultivation Base, Shandong Provincial Key Laboratory of Ophthalmology, Shandong Eye Institute, Shandong Academy of Medical Sciences, Qingdao, China

${ }^{2}$ The Affiliated Hospital of Qingdao University, Qingdao, Shandong, China Full list of author information is available at the end of the article.

Edited by I. Lavrik
}

to the development of corneal scarring and opacity ${ }^{6}$. Currently, targeting therapies primarily depend on corneal transplantation. Pharmacologic interventions with corticosteroids and mitomycin $\mathrm{C}$ are limited because of the side effects such as ulceration and reduced keratocyte density of the anterior stroma ${ }^{7-9}$.

Bromodomain-containing protein 4 (BRD4), a bromodomain and extraterminal family member and an important epigenetic reader by binding to acetylated lysines, was reported to colocalize with profibrotic transcription factors and was identified as a probable driver of fibrosis reaction ${ }^{10-12}$. From the therapeutic viewpoint, molecules that can block the fibrogenic effects of BRD4 are of great clinical interests. The prototype compound JQ1 is a small-molecule inhibitor of BRD4 that disturbs the binding of BRD4 to acetylated lysines. Recently, JQ1 was verified to attenuate various tissue fibroses, including hepatic, renal and heart fibroses, both in vivo and in vitro ${ }^{12-14}$. However, the role of JQ1 in corneal scarring remains unknown.

\section{(c) 2018 The Author(s).}

\footnotetext{
(c) Open Access This article is licensed under a Creative Commons Attribution 4.0 International License, which permits use, sharing, adaptation, distribution and reproduction cc) in any medium or format, as long as you give appropriate credit to the original author(s) and the source, provide a link to the Creative Commons license, and indicate if changes were made. The images or other third party material in this article are included in the article's Creative Commons license, unless indicated otherwise in a credit line to the material. If material is not included in the article's Creative Commons license and your intended use is not permitted by statutory regulation or exceeds the permitted use, you will need to obtain permission directly from the copyright holder. To view a copy of this license, visit http://creativecommons.org/licenses/by/4.0/.
} 
Herein, we explored the potential therapeutic effect of JQ1 on corneal scarring and corneal myofibroblast differentiation, finding that JQ1 dramatically inhibited and reversed TGF $\beta$-induced fibrotic gene expression and F-actin bundle formation in cultured human corneal fibroblasts (HCFs). Moreover, JQ1 suppressed the initial development and reversed the established corneal scarring in mice. The inhibitory effect of JQ1 on corneal scarring was related to the BRD4 inhibition and antioxidant activity.

\section{Results}

\section{JQ1 inhibits TGF $\beta$-induced corneal myofibroblast differentiation}

Primary HCFs were treated with $2 \mathrm{ng} / \mathrm{ml}$ TGF $\beta$ in the presence or absence of $200 \mathrm{nM}$ JQ1 to evaluate the inhibitory effect of JQ1 on myofibroblast differentiation. Immunofluorescence staining results showed that TGF $\beta$ treatment caused the corneal myofibroblast differentiation with enlarged morphology, enhanced staining of $\alpha$-SMA, F-actin and Collagen I. However, the JQ1 supplement suppressed the differentiation of corneal myofibroblasts induced by TGF $\beta$, which is shown as the weaker staining of $\alpha$-SMA, F-actin and Collagen I (Fig. 1a). Real-time PCR and western blot results further confirmed the inhibitory effect of JQ1 on corneal myofibroblast differentiation, including the decreased mRNA levels of $\alpha-S M A$, Fibronectin, Collagen I and SPARC, and the protein level of $\alpha$-SMA, when compared to the cells with TGF $\beta$ treatment (Fig. 1b, c).

\section{JQ1 attenuates mechanical injury-induced corneal scarring}

To investigate the effect of JQ1 during the progression of corneal scarring, a mechanical injury-induced mouse corneal scarring model was established by removing the corneal epithelium and anterior stroma. Photographs of injured corneas were captured using slit-lamp biomicroscopy with or without fluorescein staining at $1,3,5$ and 7 days post injury. The central and diskshaped scarring was remarkable at 7 days in the control group (Fig. 2a). However, JQ1-treated mice exerted alleviated corneal scarring with a significantly lower scarring score when compared to the control group at 7 days post injury $(n=12$; Fig. $2 \mathrm{~b}, \mathrm{c})$, immunofluorescence staining showed the reduced staining of $\alpha$-SMA and F-actin in JQ1-treated corneas compared to the control group (Fig. 2d), which was further confirmed by the decreased mRNA and protein expression of $\alpha$-SMA (Fig. 2e). Moreover, fluorescein staining showed the corneal epithelial healing was not impaired with the subconjunctivally injected JQ1 (Fig. 2f). The results suggest that JQ1 treatment attenuates mechanical injury-induced corneal scarring in mice, with no toxic effect on epithelial healing.

\section{JQ1 induces the dedifferentiation of corneal myofibroblasts}

To verify whether JQ1 could induce the dedifferentiation of corneal myofibroblasts in vitro, primary HCFs were treated with $2 \mathrm{ng} / \mathrm{ml}$ TGF $\beta$ for 3 and 6 days, and with TGF $\beta$ for 6 days plus JQ1 for the later 3 days, respectively (Fig. 3a). Immunofluorescence staining, realtime PCR and western blot demonstrated the complete differentiation of corneal myofibroblasts treated by TGF $\beta$ for 3 days, which assumed the identical changes with those treated by TGF $\beta$ for 6 days (Fig. 3a-c). However, the differentiated corneal myofibroblasts reversed to the fibroblastic morphology, with weaker expressions of $\alpha$-SMA, F-actin, Collagen I and SPARC, and a decreased protein level of $\alpha$-SMA after the later treatment with JQ1 for 3 days when compared to the cells solely treated with TGF $\beta$ for 3 or 6 days (Fig. 3a-c). Taken together, these results suggest that JQ1 treatment can not only inhibit myofibroblast differentiation but also induce the dedifferentiation of corneal myofibroblasts in vitro.

\section{JQ1 reverses the established corneal scarring in mice}

To further investigate whether JQ1 can reverse the established corneal scarring in vivo, the mice with corneal scarring were treated with JQ1 for 7 days from day 7 after mechanical injury, when the corneal scarring had been established (Fig. 4a). The mice with 7-day JQ1 treatment presented an alleviated corneal scarring with a significantly lower scarring score when compared to the status at 7 and 14 days post injury ( $n=12$; Fig. $4 \mathrm{~b})$. Immunofluorescence staining revealed weaker staining of $\alpha$-SMA and F-actin in the JQ1-treated corneas than the untreated groups, which was further confirmed by the decreased mRNA and protein expression of $\alpha$-SMA (Fig. 4c, d). Taken together, these results suggest that JQ1 reverses the established corneal scarring in vivo.

\section{JQ1 blocks corneal myofibroblast differentiation via BRD4 inhibition}

As JQ1 is a small-molecule inhibitor of BRD4, we hypothesized that BRD4 played an important role in modulating corneal myofibroblast differentiation. In support of this view, JQ1 administration and siRNA transfection methods were used to regulate the expression of BRD4 in telomerase-immortalized human corneal fibroblasts (HTK cell line, presented by Prof. Jester of University of California, Irvine). Following siRNA-transfection, the mRNA and protein expression of BRD4 was significantly declined and reached the same levels to JQ1 treatment (Fig. 5a, b). Similar to JQ1 administration, siRNA-mediated inhibition of BRD4 markedly reduced the mRNA level of $\alpha$-SMA, Fibronectin, Collagen $I$ and $S P A R C$, and the protein level of $\alpha$-SMA in TGF $\beta$-treated HTKs (Fig. 5c, d). These results imply that the inhibition 


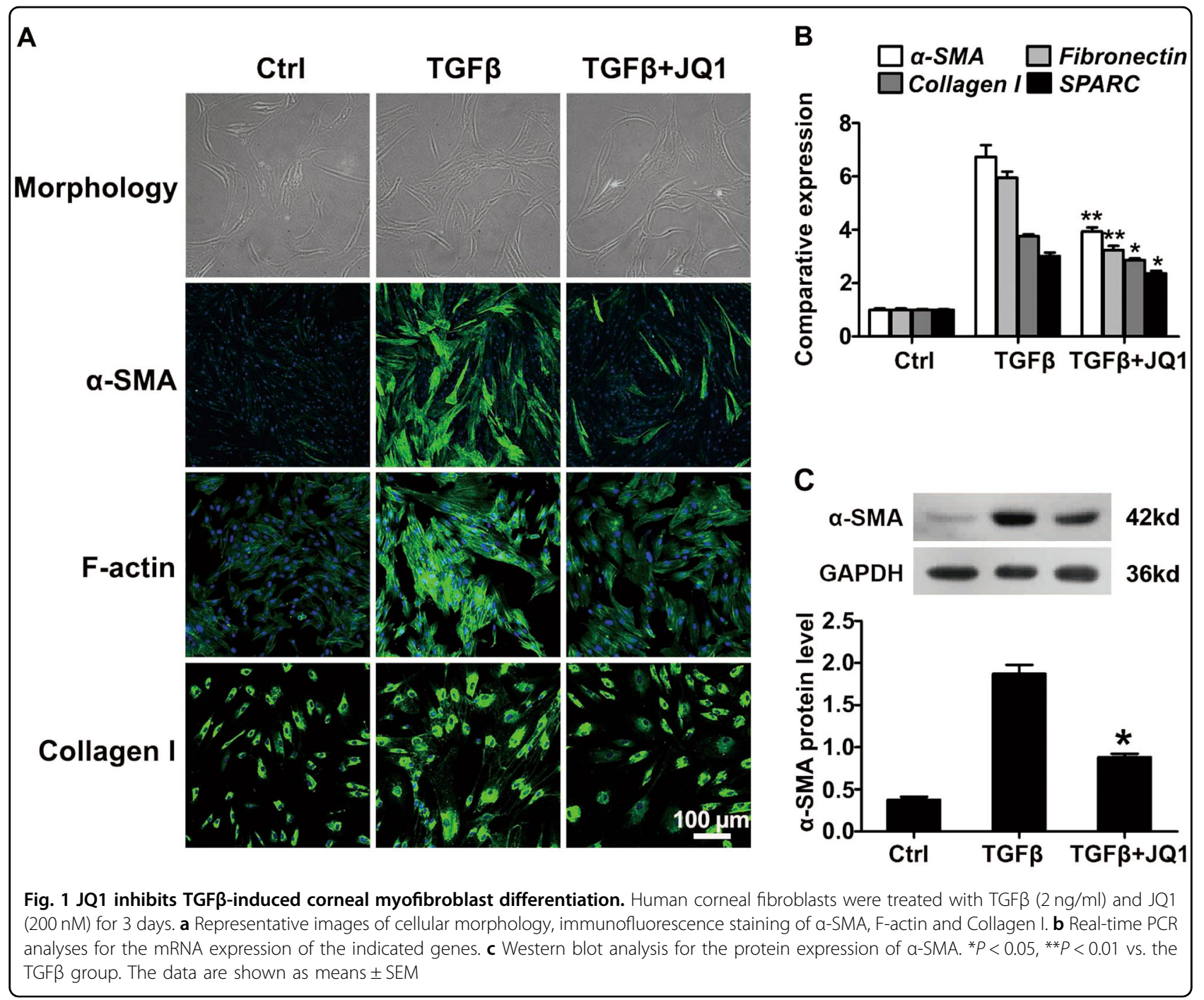

of BRD4 is an effective therapy to suppress myofibroblast differentiation.

\section{JQ1 attenuates intracellular reactive oxygen species accumulation and activates Nrf2-ARE signaling}

Previous studies have confirmed that reactive oxygen species (ROS) and Nrf2-ARE signaling played a role in corneal myofibroblast differentiation ${ }^{15,16}$. On this basis, we examined whether JQ1 can regulate the ROS accumulation induced by TGF $\beta$ treatment in corneal myofibroblast differentiation. Representative results showed weaker staining and reduced accumulation of ROS with JQ1 treatment than vehicle control in the presence of TGF $\beta$ treatment (Fig. 6a, b). Moreover, JQ1 treatment enhanced the staining of $\mathrm{Nrf} 2$ in the nucleus and promoted nuclear Nrf2 translocation, as confirmed by western blot (Fig. 6c, d). In addition, 3 days of JQ1 treatment efficiently reactivated the expression of TGF $\beta$ suppressed antioxidant genes, including NQO1 and
SOD2 (Fig. 6e). These results suggest that JQ1 promotes the accumulation of Nrf2 in the nucleus from the cytoplasm and possibly results in activating the transcription of antioxidant genes.

\section{Discussion}

As a novel therapeutic target of fibrosis, BRD4 was reported to implicate in fibrosis process by regulating fibrosis gene expression, indicating that BRD4 inhibition played an important role in the suppression of myofibroblast differentiation and organ fibrosis ${ }^{17-19}$. In our study, we demonstrated that JQ1 suppressed the TGF $\beta$-induced $\alpha$-SMA, Fibronectin, Collagen I and SPARC expressions in HCFs and prevented their differentiation to myofibroblasts, which is the initial reason of corneal scarring ${ }^{4,20}$. Then, our in vivo studies showed that subconjunctival injection of JQ1, the BRD4 inhibitor, restrained corneal scarring induced by mechanical injury in mice. We also further indicated that JQ1 


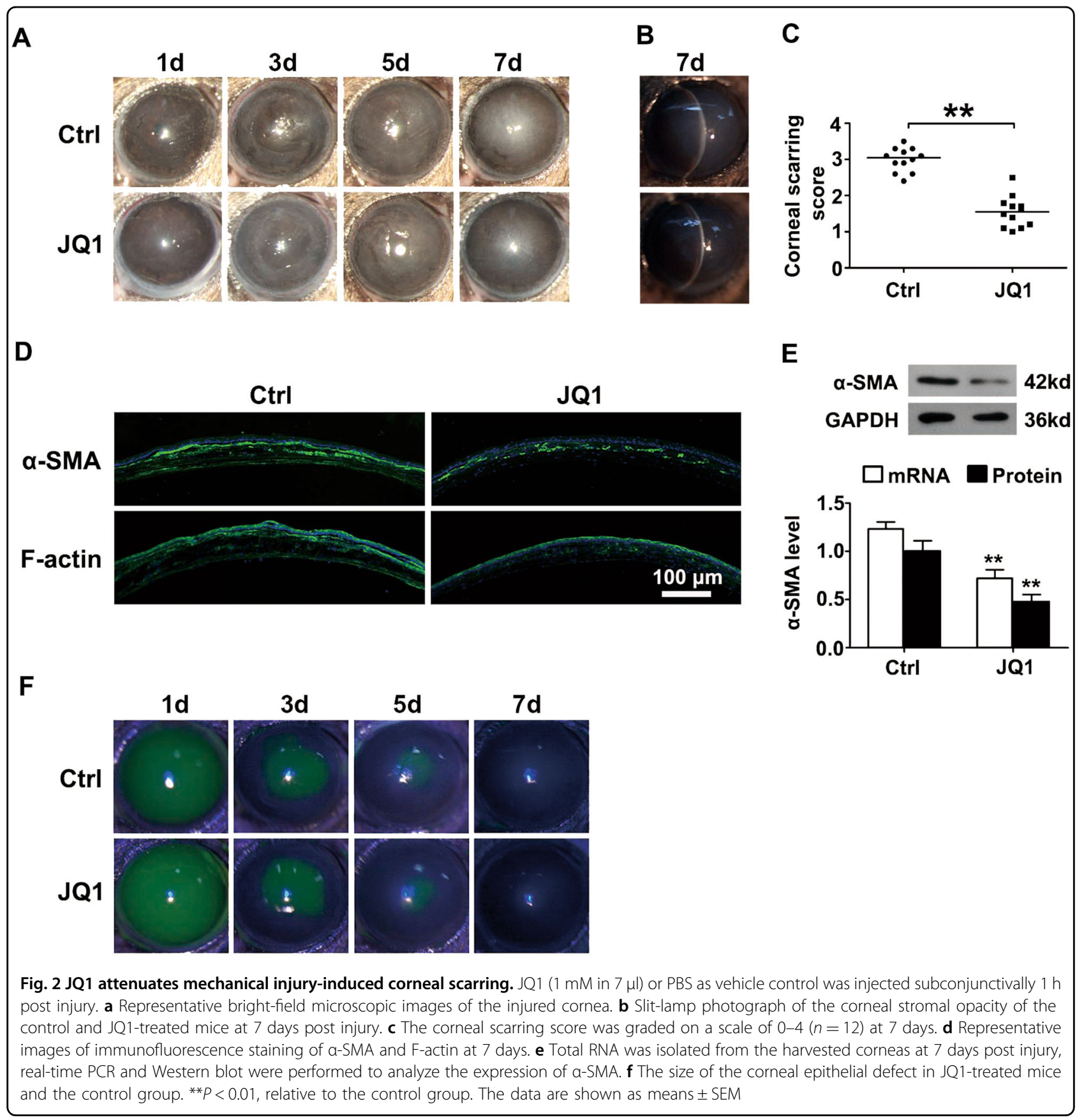

could reverse the established progression of corneal scarring in vivo and induce the dedifferentiation of myofibroblasts in vitro. Moreover, the siRNA knockdown results confirmed the role of BRD4 in suppressing TGF $\beta$-induced fibrosis gene expression in HTKs. JQ1 application was also found to alleviate TGF $\beta$ induced intracellular oxidative stress and promote the activation of Nrf2-ARE signaling in HCFs. All these results bring up the novel evidence that JQ1 suppresses and reverses corneal scarring through the regulation of BRD4 inhibition and Nrf2-dependant antioxidant induction.

Fibrosis is related to many disorders, such as chronic kidney disease, liver cirrhosis, heart failure, idiopathic pulmonary fibrosis, radiation-induced fibrosis and corneal scarring ${ }^{6,11,21-25}$. Besides the conventional therapies like keratoplasty and immunosuppressive steroids intervention, new therapeutic methods have been used to treat ocular damage caused by corneal scarring, including the inhibition of mammalian target of rapamycin (mTOR), 


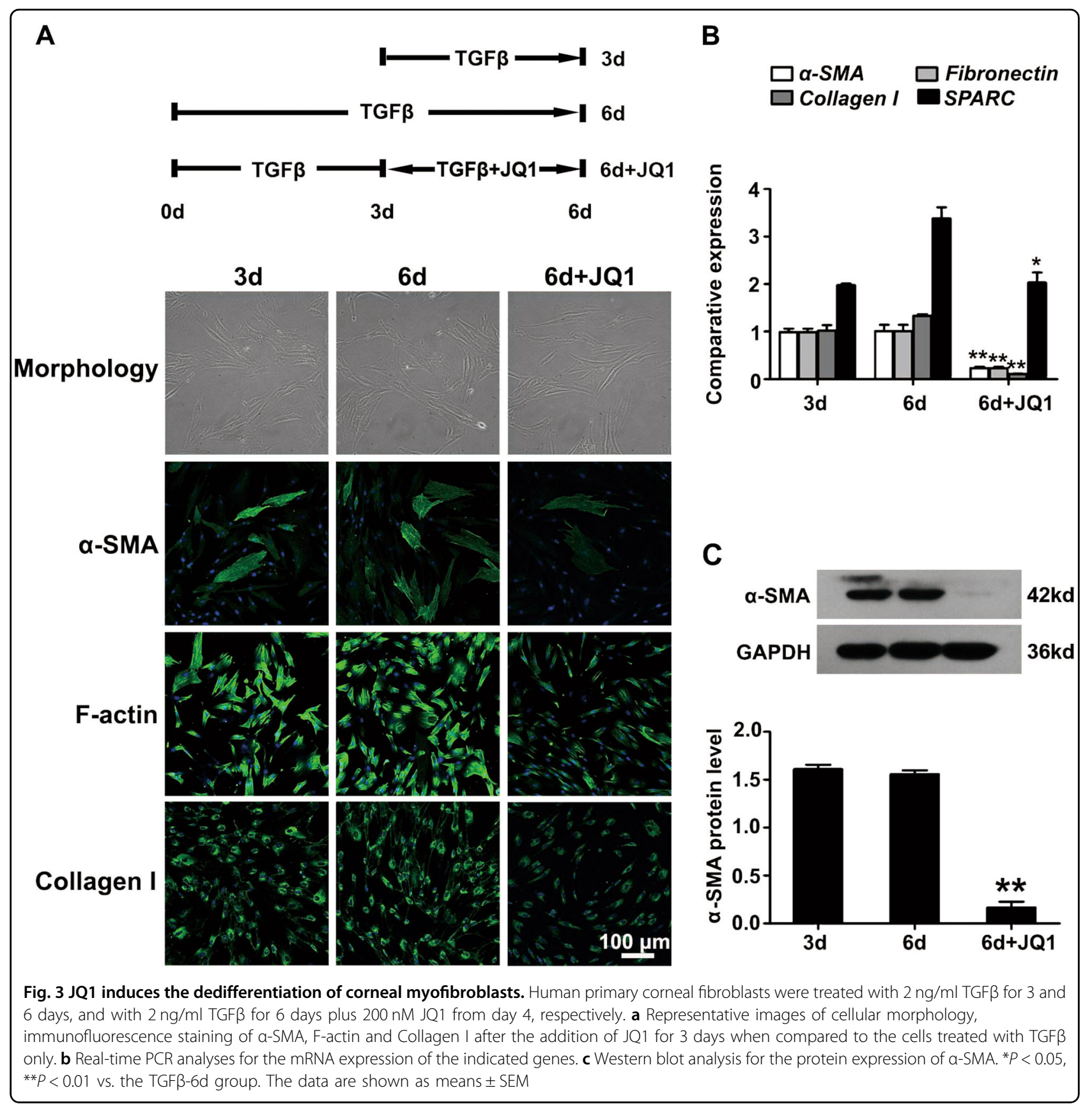

Tichostatin a, galectin-3, and the suppression of MSCs or HGF inducted by IL-1 $\beta^{9,26-28}$. Recently, BRD4 was reported to be a novel therapeutic target for various fibrosis diseases by suppressing the expression of related genes $^{29}$. JQ1, a main inhibitor of BRD4, was demonstrated to play a part in anti-fibrosis in many tissues and organs, like liver, kidney, lung and heart ${ }^{10,12-14}$, in addition to its well accepted effects in suppressing cancer proliferation, inflammation and bone destruction ${ }^{30-32}$. Our work disclosed that subconjunctivally injected JQ1 could not only suppress the initial development but also reverse the established progression of corneal scarring and myofibroblast differentiation.

Previous studies had confirmed the role of accumulated intracellular ROS induced by TGF $\beta$ in various fibrosisrelated diseases ${ }^{33-35}$. TGF $\beta$ together with its downstream NADPH oxidase 4 amplifies the oxidative stress signaling and thus enhances the fibrogenic program ${ }^{36-38}$. As an antioxidation-related transcription factor, cytoplasmic $\mathrm{Nrf} 2$ is highly unstable when it bounds to Kelch-like $\mathrm{ECH}$-associated protein 1 (Keap1) and thus results in Nrf2 selective proteasomal degradation ${ }^{39,40}$. However, 


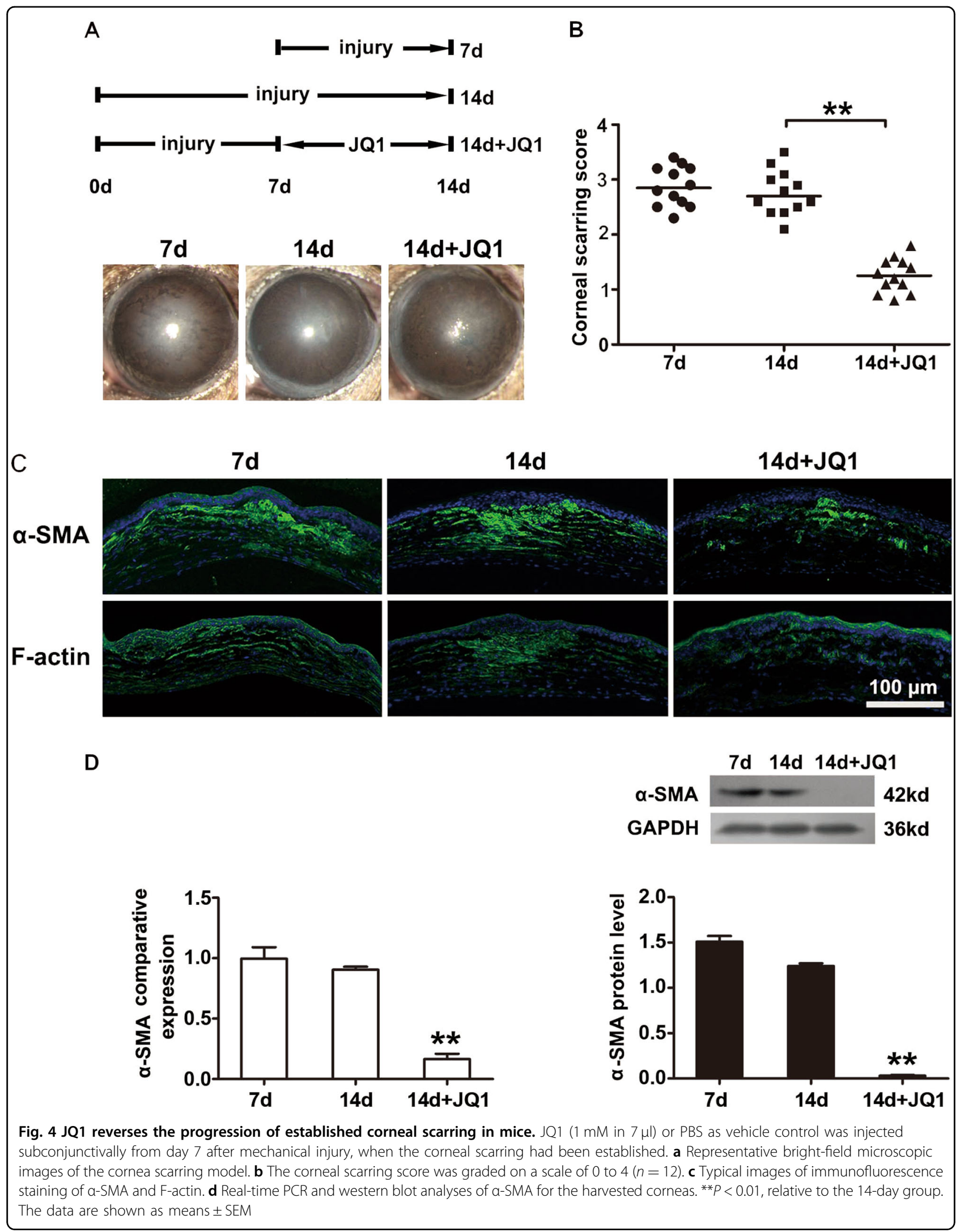



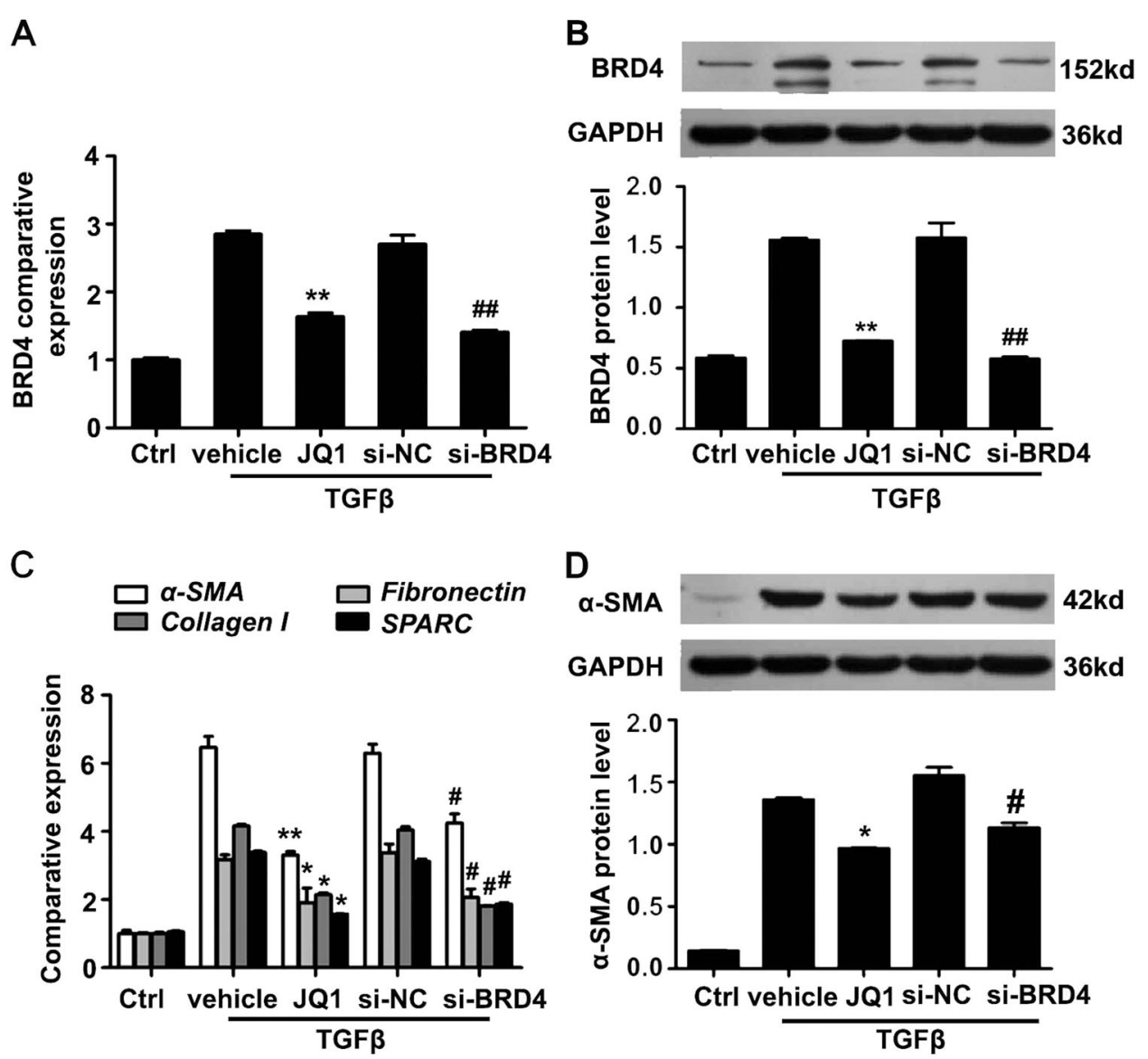

Fig. 5 JQ1 blocks corneal myofibroblast differentiation via BRD4 inhibition. Telomerase-immortalized human corneal fibroblasts (HTKs) were transfected with $1 \mu \mathrm{g}$ BRD4 siRNA or control siRNA, and treated with TGF $\beta(5 \mathrm{ng} / \mathrm{ml})$ with or without JQ1 (200 nM) for $24 \mathrm{~h}$. a Real-time PCR analysis and $\mathbf{b}$ western blot detection of the BRD4 knockdown efficiency. $\mathbf{c}$ The expression of the indicated genes after BRD4 knockdown or JQ1 administration in TGF $\beta$ treated HTKs. $\mathbf{d}$ Western blot analysis for the protein expression of a-SMA in the indicated groups. ${ }^{*} P<0.05$, ${ }^{* *} P<0.01$ vs. TGF $\beta$ group; ${ }^{P} P<0.05^{\# \#}, P<0.01$ vs. Si-NC group. Data are shown as means \pm SEM

under oxidative stress condition, Nrf2 escapes from the Nrf2-Keap1 complex and transports into the nucleus ${ }^{41,42}$, leading to an increased expression of Nrf2-ARE downstream antioxidant genes, including NADPH quinine oxidoreductase-1 (NQO1) and superoxide dismutase $(\mathrm{SOD})^{43}$. Recent evidences have revealed that BRD4 promotes the transcription of Nrf2 inhibition protein KEAP1, so that the inhibition of BRD4 by JQ1 reactivates the transcription of Nrf2-dependent genes and alleviates oxidative stress in many in vitro cell lines, like human airway smooth muscle cells, human monocytic cells, and human embryonic kidney 293 cells $^{44,45}$. Our in vitro study in HCFs further indicated that JQ1 application attenuated TGF $\beta$-induced intracellular ROS accumulation by promoting the activation of Nrf2-ARE signaling, which finally led to the inhibition of corneal myofibroblast differentiation. Our findings bring forward a novel working model of JQ1 on anti-fibrosis partly through the activation of Nrf2-dependent anti-ROS pathway in HCFs, which might serve as the potential mechanism of JQ1 treatment of corneal scarring.

In conclusion, we identified that JQ1 administration not only inhibited the myofibroblastic differentiation and corneal scarring formation, but also induced myofibroblastic dedifferentiation and established corneal scarring attenuation. The downstream Nrf2-ARE signaling was implicated in mediating the effect of JQ1 on suppressing and reversing mechanical injury-induced corneal scarring. Therefore, our study indicates that BRD4 is a potential therapeutic target for corneal fibrosis, and JQ1 intervention serves as an attractive therapy for corneal haze or scar resolution.

\section{Materials and methods \\ Animals}

Male C57BL/6 mice (6-8 weeks) were purchased from the Beijing Pharmacology Institute (Beijing, China) and maintained in the animal center of Shandong Eye 

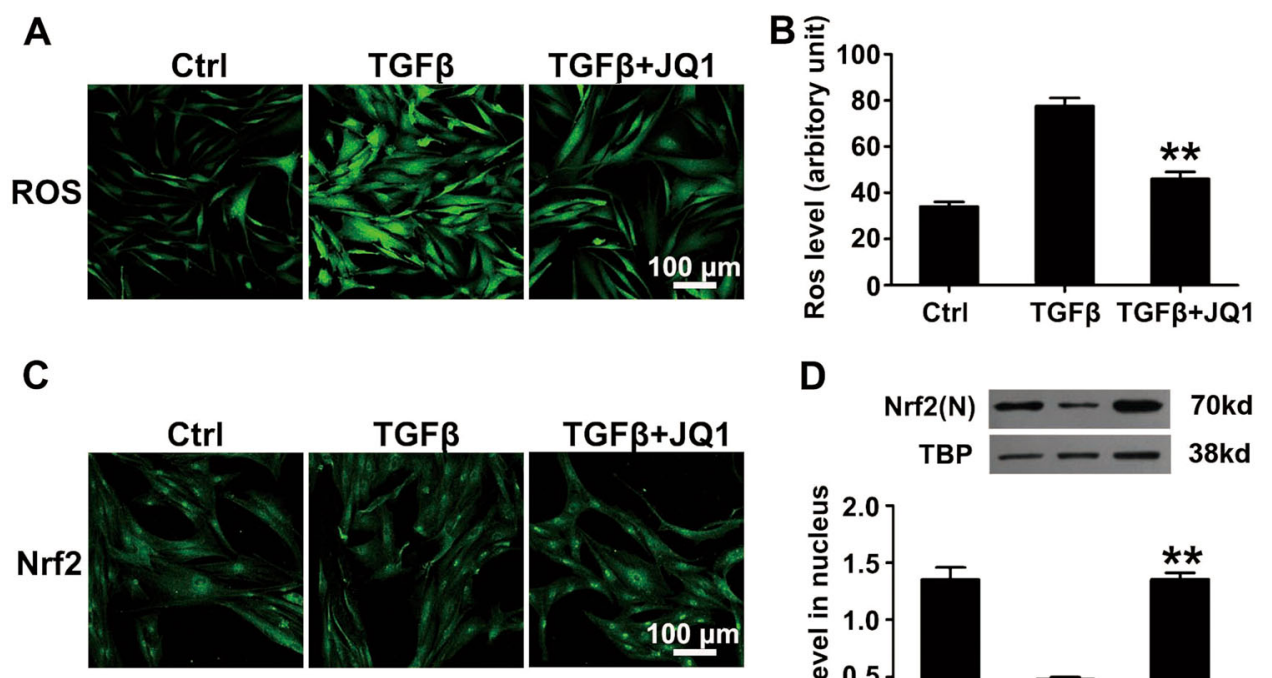

E
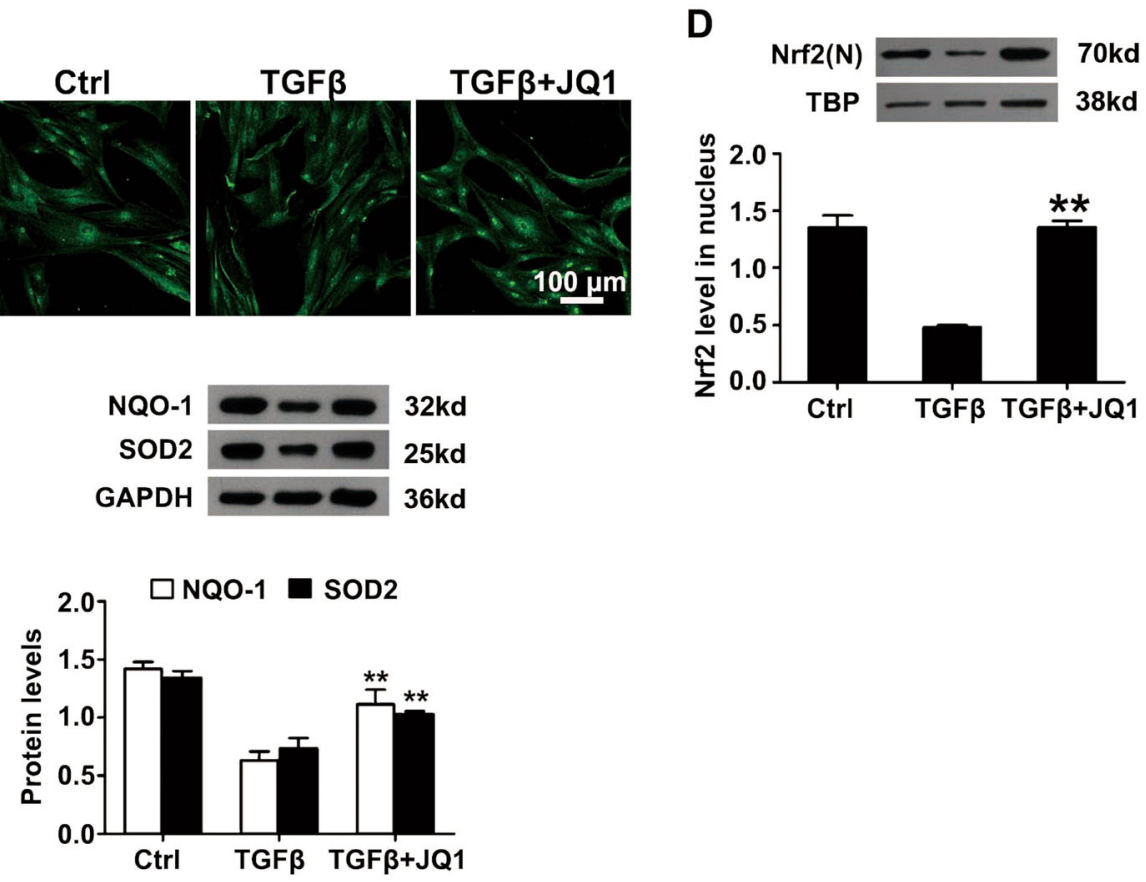

Fig. 6 JQ1 attenuates intracellular ROS accumulation and activates Nrf2-ARE signaling. Human corneal fibroblasts were treated with TGF $\beta$ $(2 \mathrm{ng} / \mathrm{ml})$ and JQ1 $(200 \mathrm{nM})$ for 3 days. a ROS staining in corneal fibroblasts treated with TGF $\beta$ and JQ1. b Absorbance measurement of ROS in the indicated groups. $\mathbf{c}$ Nuclear accumulation of Nrf2 based on immunofluorescence staining. $\mathbf{d}$ The Nrf2 level in the nucleus based on the Western blot analysis. e The expression of the Nrf2-ARE downstream antioxidant protein including NQO1 and SOD2 in the indicated groups. Nrf2 (N) indicates Nrf2 in the nucleus. ${ }^{*} P<0.05,{ }^{*} P<0.01$ vs. TGF $\beta$ group. Data are shown as means \pm SEM

Institute. All animal experiments were performed in accordance with the ARVO Statement for the Use of Animals in Ophthalmic and Vision Research and approved by the Institutional Review Board of Shandong Eye Institute. The corneal scarring model was prepared according to previous reports with minor modifications $^{28,46}$. In brief, under systemic and topical anesthesia, the mouse corneal epithelium ( $3 \mathrm{~mm}$ in diameter) and anterior stromal portion were removed with the algerbrush II corneal rust ring remover (Alger Co, Lago Vista, TX, USA) and a razor blade, respectively. JQ1 ( $1 \mathrm{mM}$ in $7 \mu \mathrm{l}$; Abcam, Cambridge, MA, USA) or PBS as vehicle control was injected subconjunctivally $1 \mathrm{~h}$ post injury. Finally, the mice were topically administered with ofoxacin eye drops (Santen, Osaka, Japan) to avoid infection. Only one eye was used in each animal for all experiments. All eyes were photographed under slit lamp (BQ900; Haag-Streit, Bern, Switzerland) at 1, 3, 5 and 7 days after the mechanical injury for the grading of corneal scarring $(n=12)$. The corneal scarring was graded on a scale of $0-4$ (0, completely clear; 0.5 , minimal scarring with a careful oblique illumination; 1 , mild scarring not interfering with visibility of fine iris details; 2 , mild opacification of iris details; 3 , moderate opacification of the iris and lens; 4, complete opacification of the anterior chamber and iris) ${ }^{26}$. To evaluate the potential toxic effect of JQ1 on corneal epithelial regeneration, $0.25 \%$ fluorescein sodium was used to stain the defects of corneal epithelium, and photographs were taken at 1, 3, 5, 7 days after injury. Mouse corneas were collected for real-time PCR, immunofluorescence staining and Western blot analysis.

\section{Cell culture}

Human corneal fibroblasts (HCFs) were isolated and cultured as previously reported ${ }^{4}$. To evaluate the 
Table 1 Nucleotide sequences of primers used for RT-qPCR

\begin{tabular}{|c|c|c|c|}
\hline Genes & Forward primer $\left(5^{\prime}-3^{\prime}\right)$ & Reverse primer $\left(5^{\prime}-3^{\prime}\right)$ & Gene accession \\
\hline h- $a-S M A$ & GGTGACGAAGCACAGAGCAA & CAGGGTGGGATGCTCTTCAG & NM_001141945.2 \\
\hline h-fibronectin & GGGACCGTCAGGGAGAAAA & CGAGATATTCCTTCTGCCACTGTT & NM_212482.2 \\
\hline h-collagen I & TTGTGCGATGACGTGATCTGT & TTGGTCGGTGGGTGACTCTG & NM_000088.3 \\
\hline h-SPARC & GGCTTCTCCTCCTCTGTCTT & AACCGATTCACCAACTCCAC & NM_003118.3 \\
\hline h-BRD4 & CTAAACTGGAGGCCCGTGAGT & CAAAGCGCATTTCGAACACA & NM_014299.2 \\
\hline h-GAPDH & ATGCTGGCGCTGAGTACGT & AGCCCCAGCCTTCTCCAT & NM_002046.4 \\
\hline$m-a-S M A$ & TGCCGAGCGTGAGATTGTC & CGTTCGTTCCAATGGTGATC & NM_007392.3 \\
\hline $\mathrm{m}-\mathrm{GAPDH}$ & GATGCCCCCATGTTTGTGAT & GGCATGGACTGTGGTCATGAG & NM_008084.2 \\
\hline
\end{tabular}

inhibitory effect of JQ1 in myofibroblast differentiation, HCFs were treated with $2 \mathrm{ng} / \mathrm{ml}$ TGF $\beta$ (R\&D Systems, Minneapolis, MN, USA) for 3 days with or without $200 \mathrm{nM}$ JQ1. To evaluate the reversal effect of JQ1 on corneal myofibroblasts, HCFs were treated with $2 \mathrm{ng} / \mathrm{ml}$ TGF $\beta$ for 3 days followed by $200 \mathrm{nM}$ JQ1 treatment for additional 3 days. All cells were further analyzed for $\alpha$-SMA, Fibronectin, Collagen I, and SPCRC expressions, F-actin bundle formation, ROS accumulation, Nrf2 and Nrf2-ARE downstream antioxidant gene expressions. The concentrations of TGF $\beta$ and JQ1 were optimized according to our preliminary experiment.

\section{Transfection of siRNAs}

Telomerase-immortalized human corneal fibroblasts (HTK cell line) were cultured as previously described ${ }^{43}$. HTKs $\left(2 \times 10^{5}\right.$ cells) were seeded and transfected with $1 \mu \mathrm{g}$ BRD4 siRNA (sc-43639; Santa Cruz Biotechnology, Santa Cruz, CA, USA) or control siRNA duplex (sc-37007; Santa Cruz Biotechnology). After 5-7 h incubation, the transfection medium was replaced with normal medium for $40 \mathrm{~h}$, the cells were treated with $5 \mathrm{ng} / \mathrm{ml}$ TGF $\beta$ with or without $200 \mathrm{nM}$ JQ1 for $24 \mathrm{~h}$. Then, the cells were collected for the extraction of total RNA and total proteins. The expressions of BRD4 and fibrotic genes were detected at both the mRNA and protein levels.

\section{Real-time qPCR}

Total RNA was extracted from corneal fibroblasts or corneal tissues using the NucleoSpin RNA kit (BD Biosciences, Palo Alto, CA, USA). Isolated RNA was reversely transcribed into cDNA using the PrimeScript first-strand cDNA synthesis kit (TaKaRa, Dalian, China). Real-time PCR was carried out using SYBR Green reagents and the Applied Biosystems 7500 Real-Time PCR System (Applied Biosystems, Foster City, CA, USA). The specific primers used in this assay are listed in Table 1. The results were analyzed with the Sequence Detection System (SDS) software (Applied Biosystems) using GAPDH as an internal control.

\section{Immunofluorescence staining}

Cultured cells were fixed with $4 \%$ paraformaldehyde for $10 \mathrm{~min}$ at room temperature. Mouse corneal cryosections were fixed with ice methanol for $10 \mathrm{~min}$ at $-20^{\circ} \mathrm{C}$. All samples were blocked with 5\% BSA (Sigma-Aldrich Corp, St. Louis, MO, USA) and incubated with FITC-conjugated phalloidin (Alexis Biochemicals, San Diego, CA, USA), rabbit anti- $\alpha$-SMA (Abcam), rabbit anti-Collagen I (Abcam) and rabbit anti-Nrf2 (Abcam) antibodies at $4{ }^{\circ} \mathrm{C}$ overnight. The samples were subsequently incubated with Alexa Fluor 488-labeled donkey anti-rabbit IgG antibody (Invitrogen, Carlsbad, CA, USA) at $37^{\circ} \mathrm{C}$ for $1 \mathrm{~h}$. Nuclear counterstaining was performed using 4',6-diamidino-2-phenylindole (DAPI, Sigma-Aldrich). Finally, the staining was observed under a confocal laser-scanning microscope (Nikon, Tokyo, Japan) or a fluorescence microscope (Nikon).

\section{Western blot analysis}

Cultured cells or mouse corneal tissues were lysed with RIPA buffer containing protease inhibitors (Beyotime, Jiangsu, China). The protein samples were separated on SDS polyacrylamide gels and transferred to PVDF membranes (Millipore, Billerica, MA, USA). The blots were blocked by non-fat dry milk for at least $1 \mathrm{~h}$, and incubated with rabbit anti- $\alpha$-SMA (Abcam), rabbit anti-BRD4 (Abcam), rabbit anti-Nrf2 (Abcam), mouse anti-TBP (Abcam), rabbit anti-SOD2 (Abcam) and rabbit antiNQO1 (Abcam) overnight at $4{ }^{\circ} \mathrm{C}$. After incubation with an HRP-conjugated secondary antibody (Zsbio, Beijing, China), the Western blots were visualized using the enzyme-linked chemiluminescence kit (Chemicon, Temecula, CA, USA). The data analysis was performed using the Image J Software (NIH, Bethesda, MD, USA) to quantify the levels of proteins. 


\section{Measurement of intracellular reactive oxygen species generation}

For ROS staining, the cultured HCFs were washed with serum-free medium and incubated with $10 \mu \mathrm{M}$ fluorescence probe 2,7-dichlorodihydrofluorescein diacetate, acetyl ester (DCHF-DA; Molecular Probes, Eugene, OR, USA) for $30 \mathrm{~min}$ at $37^{\circ} \mathrm{C}$, the unlabeled dye was washed by serum-free medium and the cells were observed under a Nikon confocal microscope. For the measurement of ROS generation, the cells were harvested and incubated with $5 \mu \mathrm{M}$ DCHF-DA for $20 \mathrm{~min}$, and then the fluorescence intensity was measured by a multimode microplate reader (SpectraMax M2; Molecular Devices, Menlo Park, CA, USA).

\section{Statistical analysis}

The figures in this study are representative of more than 3 different experiments. Corneal injury and scarring were performed in a masked fashion. Twelve images were used for in vivo quantification in each group. Differences between the control and treated groups were analyzed using the Student's $t$ test. $P<0.05$ was considered to be statistically significant.

\section{Acknowledgements}

This work was partially supported by the Shandong Provincial Natural Science Fund for Distinguished Young Scholars (JQ201518) and the National Natural Science Foundation of China (81400383, 81530027, 81470610). M.Q. is supported by the Shandong Provincial Key Research and Development Program (2018GSF118204).

\section{Author details}

${ }^{1}$ State Key Laboratory Cultivation Base, Shandong Provincial Key Laboratory of Ophthalmology, Shandong Eye Institute, Shandong Academy of Medical Sciences, Qingdao, China. ${ }^{2}$ The Affiliated Hospital of Qingdao University, Qingdao, Shandong, China. ${ }^{3}$ School of Medicine and Life Sciences, University of Jinan-Shandong Academy of Medical Sciences, Jinan, Shandong, China

\section{Author contributions}

Q.Z. and M.Q. designed the experiments and analyzed the data. M.Q., X.Z., M.D., X.P. and J.B. performed the experiments. X.Z. and M.D. performed animal surgeries. M.Q. and X.H. wrote the manuscript with guidance and revisions from Q.Z. All other authors declare that they have no competing interests.

\section{Conflict of interest}

The authors declare that they have no conflict of interest.

\section{Publisher's note}

Springer Nature remains neutral with regard to jurisdictional claims in published maps and institutional affiliations.

Received: 14 March 2018 Revised: 21 May 2018 Accepted: 3 June 2018 Published online: 28 June 2018

\section{References}

1. Karamichos, D., Guo, X. Q., Hutcheon, A. E. \& Zieske, J. D. Human corneal fibrosis: an in vitro model. Invest. Ophthalmol. Vis. Sci. 51, 1382-1388 (2010).

2. Jester, J. V., Barry-Lane, P. A., Petroll, W. M., Olsen, D. R. \& Cavanagh, H. D. Inhibition of corneal fibrosis by topical application of blocking antibodies to TGF-beta in the rabbit. Cornea 16, 177-187 (1997).
3. Torricelli, A. A., Santhanam, A., Wu, J., Singh, V. \& Wilson, S. E. The corneal fibrosis response to epithelial-stromal injury. Exp. Eye Res. 142, 110-118 (2016).

4. Zhou, Q. et al. TGFbeta mediated transition of corneal fibroblasts from a proinflammatory state to a profibrotic state through modulation of histone acetylation. J. Cell. Physiol. 224, 135-143 (2010).

5. Nelson, E. F., Huang, C. W., Ewel, J. M., Chang, A. A. \& Yuan, C. Halofuginone down-regulates Smad3 expression and inhibits the TGFbeta-induced expression of fibrotic markers in human corneal fibroblasts. Mol. Vis. 18, 479-487 (2012).

6. Shu, D. Y. \& Lovicu, F. J. Myofibroblast transdifferentiation: The dark force in ocular wound healing and fibrosis. Prog. Retin. Eye. Res. 60 44-65 (2017).

7. Dougherty, P. J., Hardten, D. R. \& Lindstrom, R. L. Corneoscleral melt after pterygium surgery using a single intraoperative application of mitomycin-C. Cornea 15, 537-540 (1996).

8. Netto, M. V. et al. Effect of prophylactic and therapeutic mitomycin C on corneal apoptosis, cellular proliferation, haze, and long-term keratocyte density in rabbits. J. Refract. Surg. 22, 562-574 (2006).

9. Milani, B. Y. et al. Rapamycin inhibits the production of myofibroblasts and reduces corneal scarring after photorefractive keratectomy. Invest. Ophthalmol. Vis. Sci. 54, 7424-7430 (2013).

10. Ding, N. et al. BRD4 is a novel therapeutic target for liver fibrosis. Proc. Natl Acad. Sci. USA 112, 15713-15718 (2015).

11. Xiong, $C$. et al. Pharmacological targeting of BET proteins inhibits renal fibroblast activation and alleviates renal fibrosis. Oncotarget 7, 69291-69308 (2016).

12. Tang, $X$. et al. BET bromodomain proteins mediate downstream signaling events following growth factor stimulation in human lung fibroblasts and are involved in bleomycin-induced pulmonary fibrosis. Mol. Pharmacol. 83, 283-293 (2013).

13. Zhou, B. et al. Brd4 inhibition attenuates unilateral ureteral obstructioninduced fibrosis by blocking TGF-beta-mediated Nox4 expression. Redox Biol. 11, 390-402 (2017).

14. Duan, Q. et al. BET bromodomain inhibition suppresses innate inflammatory and profibrotic transcriptional networks in heart failure. Sci. Transl. Med. 9 , (2017). 28515341.

15. Hecker, L. et al. Reversal of persistent fibrosis in aging by targeting Nox4-Nrf2 redox imbalance. Sci. Transl. Med 6, 231 ra247 (2014).

16. Dong, J. \& Ma, Q. Suppression of basal and carbon nanotube-induced oxidative stress, inflammation and fibrosis in mouse lungs by Nrf2. Nanotoxicology 10, 699-709 (2016).

17. Kumar, K. et al. BET inhibitors block pancreatic stellate cell collagen I production and attenuate fibrosis in vivo. JCl Insight 2, e88032 (2017).

18. Zhubanchaliyev, A., Temirbekuly, A., Kongrtay, K., Wanshura, L. C. \& Kunz, J. Targeting mechanotransduction at the transcriptional level: YAP and BRD4 are novel therapeutic targets for the reversal of liver fibrosis. Front. Pharmacol. 7, 462 (2016).

19. Filippakopoulos, P. et al. Selective inhibition of BET bromodomains. Nature 468, 1067-1073 (2010).

20. Jester, J. V., Petroll, W. M. \& Cavanagh, H. D. Corneal stromal wound healing in refractive surgery: the role of myofibroblasts. Prog. Retin. Eye. Res. 18, 311-356 (1999).

21. Wynn, T. A. Cellular and molecular mechanisms of fibrosis. J. Pathol. 214 199-210 (2008).

22. Huang, X. R., Chung, A. C., Wang, X. J., Lai, K. N. \& Lan, H. Y. Mice overexpressing latent TGF-beta1 are protected against renal fibrosis in obstructive kidney disease. Am. J. Physiol. Ren. Physiol. 295, F118-F127 (2008).

23. Kanisicak, O. et al. Genetic lineage tracing defines myofibroblast origin and function in the injured heart. Nat. Commun. 7, 12260 (2016).

24. Valinciute, G. et al. BET-bromodomain inhibitors modulate epigenetic patterns at the diacylglycerol kinase alpha enhancer associated with radiation-induced fibrosis. Radiother. Oncol. 125, 168-174 (2017).

25. Zeybel, M. et al. A proof-of-concept for epigenetic therapy of tissue fibrosis: inhibition of liver fibrosis progression by 3-deazaneplanocin A. Mol. Ther. 25, 218-231 (2017).

26. Sharma, A., Mehan, M. M., Sinha, S., Cowden, J. W. \& Mohan, R. R. Trichostatin a inhibits corneal haze in vitro and in vivo. Invest. Ophthalmol. Vis. Sci. 50, 2695-2701 (2009).

27. Chen, W. S., Cao, Z., Leffler, H., Nilsson, U. J. \& Panjwani, N. Galectin-3 Inhibition by a Small-Molecule Inhibitor Reduces Both Pathological Corneal Neovascularization and Fibrosis. Invest. Ophthalmol. Vis. Sci. 58, 9-20 (2017). 
28. Mittal, S. K. et al. Restoration of corneal transparency by mesenchymal stem cells. Stem Cell Rep. 7, 583-590 (2016).

29. Stratton, M. S., Haldar, S. M., McKinsey, T. A. BRD4 inhibition for the treatment of pathological organ fibrosis. F1000Res. 6, (2017) 28721198

30. Lee, D. H. et al. Synergistic effect of JQ1 and rapamycin for treatment of human osteosarcoma. Int. J. Cancer 136, 2055-2064 (2015).

31. Meng, S. et al. BET Inhibitor JQ1 blocks inflammation and bone destruction. J. Dent. Res. 93, 657-662 (2014).

32. Shao, Q. et al. BET protein inhibitor JQ1 attenuates Myc-amplified MCC tumor growth in vivo. Cancer Res. 74, 7090-7102 (2014).

33. Richter, K., Konzack, A., Pihlajaniemi, T., Heljasvaara, R. \& Kietzmann, T. Redoxfibrosis: Impact of TGFbeta1 on ROS generators, mediators and functional consequences. Redox Biol. 6, 344-352 (2015).

34. Samarakoon, R., Overstreet, J. M. \& Higgins, P. J. TGF-beta signaling in tissue fibrosis: redox controls, target genes and therapeutic opportunities. Cell Signal. 25, 264-268 (2013).

35. Liu, R. M. \& Desai, L. P. Reciprocal regulation of TGF-beta and reactive oxygen species: a perverse cycle for fibrosis. Redox Biol. 6, 565-577 (2015).

36. Amara, N. et al. NOX4/NADPH oxidase expression is increased in pulmonary fibroblasts from patients with idiopathic pulmonary fibrosis and mediates TGFbeta1-induced fibroblast differentiation into myofibroblasts. Thorax $\mathbf{6 5}$, 733-738 (2010).

37. Hecker, L. et al. NADPH oxidase-4 mediates myofibroblast activation and fibrogenic responses to lung injury. Nat. Med. 15, 1077-1081 (2009).
38. Martin-Garrido, A. et al. NADPH oxidase 4 mediates TGF-beta-induced smooth muscle alpha-actin via p38MAPK and serum response factor. Free Radic. Biol. Med. 50, 354-362 (2011).

39. Suzuki, T. \& Yamamoto, M. Molecular basis of the Keap1-Nrf2 system. Free Radic. Biol. Med. 88, 93-100 (2015).

40. McMahon, M. Itoh, K. Yamamoto, M. \& Hayes, J. D. Keap1-dependent proteasomal degradation of transcription factor $\mathrm{Nrf} 2$ contributes to the negative regulation of antioxidant response element-driven gene expression. J. Biol. Chem. 278, 21592-21600 (2003).

41. Vriend, J. \& Reiter, R. J. The Keap1-Nrf2-antioxidant response element pathway: a review of its regulation by melatonin and the proteasome. Mol. Cell Endocrinol. 401, 213-220 (2015).

42. Kovac, S. et al. Nif2 regulates ROS production by mitochondria and NADPH oxidase. Biochim. Biophys. Acta 1850, 794-801 (2015).

43. Yang, L. et al. Trichostatin A inhibits transforming growth factor-beta-induced reactive oxygen species accumulation and myofibroblast differentiation via enhanced NF-E2-related factor 2-antioxidant response element signaling. Mol. Pharmacol. 83, 671-680 (2013).

44. Michaeloudes, C. et al. Bromodomain and extraterminal proteins suppress NFE2-related factor 2-mediated antioxidant gene expression. J. Immunol. 192, 4913-4920 (2014).

45. Hussong, $M$. et al. The bromodomain protein BRD4 regulates the KEAP1/ NRF2-dependent oxidative stress response. Cell Death Dis. 5, e1195 (2014).

46. Rittie, L., Hutcheon, A. E. K. \& Zieske, J. D. Mouse models of corneal scarring Methods Mol. Biol. 1627, 117-122 (2017). 